\title{
Sarcopenia in Korea: Prevalence and Clinical Aspects
}

\author{
Kyoung Min Kim, MD ${ }^{1, *}$, Soo Lim, $\mathrm{MD}^{1, *}$, Kyung Mook Choi, $\mathrm{MD}^{2}$, Jung Hee Kim, $\mathrm{MD}^{3}$, \\ Sung Hoon Yu, MD ${ }^{4}$, Tae Nyun Kim, MD ${ }^{5}$, Wook Song, PhD ${ }^{6}$, Jae-Young Lim, MD ${ }^{7}$, \\ Chang Won Won, $\mathrm{MD}^{8}$, Hyung Joon Yoo, $\mathrm{MD}^{4}$, Hak Chul Jang, MD ${ }^{1}$; \\ Sarcopenia Study Group of Korean Geriatrics Society \\ ${ }^{I}$ Department of Internal Medicine, Seoul National University Bundang Hospital, Seoul National University \\ College of Medicine, Seongnam, \\ ${ }^{2}$ Department of Internal Medicine, Korea University College of Medicine, Seoul, \\ ${ }^{3}$ Department of Internal Medicine, Seoul National University Hospital, Seoul National University College of Medicine, Seoul, \\ ${ }^{4}$ Department of Internal Medicine, Hallym University Kangnam Sacred Hospital, Seoul, \\ ${ }^{5}$ Department of Internal Medicine, Inje University College of Medicine, Busan, \\ ${ }^{6}$ Health and Exercise Science Laboratory, Institute of Sports Science, Department of Physical Education, \\ Seoul National University, Seoul, \\ ${ }^{7}$ Department of Rehabilitation Medicine, Seoul National University Bundang Hospital, Seoul National University \\ College of Medicine, Seongnam, \\ ${ }^{8}$ Department of Family Medicine, Kyung Hee University College of Medicine, Seoul, Korea
}

Sarcopenia has been defined as the considerable loss of skeletal muscle mass and strength that results in frailty in the elderly. Because muscle tissue plays diverse important roles in human, sarcopenia presents many negative health-related consequences including impaired energy homeostasis, falls and cardiovascular disease, and subsequently higher mortality. It is becoming evident that sarcopenia has a negative impact on the healthy life of the elderly. The European Working Group on Sarcopenia, the International Working Group on Sarcopenia and the Asian Working Group on Sarcopenia have released position statement regarding sarcopenia, and more recently the Foundation for the National Institutes of Health Sarcopenia Project has provided a new guideline for assessment of sarcopenia. At this time, there have been several data delineating the status of sarcopenia in Korea. This review focuses on largescale cohorts that assessed sarcopenia and highlights the controversies surrounding the clinical definition and prevalence of sarcopenia in Korea.

Key Words: Sarcopenia, Frailty, Elderly, Appendicular lean mass

\section{INTRODUCTION}

A progressive decrease in muscle mass and an in-

\footnotetext{
- Received: March 10, 2015 > Revised: March 20, 2015

- Accepted: March 23, 2015

Address for correspondence: Hak Chul Jang, MD, $\mathrm{PhD}$

Department of Internal Medicine, Seoul National University

Bundang Hospital, Seoul National University College of Medicine,

82 Gumi-ro 173 beon-gil, Bundang-gu, Seongnam 463-707, Korea

Tel: +82-31-787-7005, Fax: +82-31-787-4052

E-mail: janghak@snu.ac.kr

*These authors contributed equally to this study and should be considered co-first authors.
}

crease in fat mass are typical body compositional changes associated with aging in humans. In these age-related body compositional changes, sarcopenia is defined as a condition characterized by considerable loss of muscle mass and strength, decreased physical performance, and consequent frailty in the elderly ${ }^{1}$. Muscle tissue plays various important roles in the human body; thus, loss of muscle mass can cause diverse functional and metabolic derangements in the elderly. Because of the clinical importance of sarcopenia in the elderly, the number of studies focusing the prevalence, etiology, and clinical issue of sarcopenia has been increasing in many countries, including Korea ${ }^{2-4)}$. Moreover, consensus papers about defi- 
nition, prevalence, and clinical importance of sarcopenia have been published by various working groups, including the International Working Group on Sarcopenia (IWGS), the European Working Group on Sarcopenia in Older People (EWGSOP), and Asian Working Group for Sarcopenia ${ }^{5-7)}$.

Appendicular skeletal muscle mass (ASM) divided by height squared (ASM/ht ${ }^{2}$ ) or by weight (ASM/wt) are representative methods used for the definition of sarcopenia $^{8,9)}$. When determining cutoff values for sarcopenia, a mix of criteria of less than 1 standard deviation (SD) or 2 SDs below the mean values of healthy adults, or the lowest quintile of study populations has been used ${ }^{10,11}$. However, the prevalence and associations with other clinical parameters could be described differently according to the methods used to categorize sarcopenia ${ }^{12-15)}$.

Korea is one of the fastest aging countries, and it has become increasingly evident that sarcopenic status in the elderly is closely related to many clinical consequences including functional limitations, metabolic impairment, and increased cardiovascular risk. This review aims to introduce the representative cohorts for assessing sarcopenia in Korea and their published data, and will highlight the controversies surrounding the definition and prevalence of sarcopenia in Korea.

\section{OPERATIONAL DEFINITION OF SARCOPENIA}

The diagnostic criterion of sarcopenia is mainly based on muscle mass. Baumgartner et al.") first suggested a height squared adjusted ASM index in the New Mexico Elder Health Survey. They also showed a significant association between physical disabilities and sarcopenia defined by that that index. However, the relationships were relatively weak for women. This definition was criticized because of the significant positive correlation with body mass index (BMI), suggesting that subjects with greater BMI having a larger amount of fat, are less likely to classified as having sarcopenia ${ }^{16)}$. In this respect, Newman et al. ${ }^{16)}$ suggested an alternative definition adjusted for both height and fat mass, but this method has not been widely used because it is difficult to apply practically. In 2002, Janssen et al. ${ }^{8)}$ presented another method. They defined relative muscle mass index as skeletal muscle mass, which was derived from bioimpedance analysis, divided by weight. In other words, absolute skeletal muscle mass $(\mathrm{kg})$ was converted to percentage skeletal muscle mass (skeletal muscle mass/ body weight $\times 100$ ) and termed the skeletal muscle index (SMI). Since Janssen et al. ${ }^{8)}$ proposed their method, "ASM/ wt" modified by the index of Janssen et al. ${ }^{8)}$, which is derived from dual energy X-ray absorptiometry (DEXA), has been also used as an alternative method together with the index described by Baumgartner et al. ${ }^{17)}$.

\section{COHORT STUDIES OF SARCOPENIA IN KOREA}

\section{Studies in the Korean National Health and Nutrition Examination Survey}

The Korean National Health and Nutrition Examination Survey (KNHANES) is a nationwide, population-based, crosssectional study and has been conducted periodically since 1998 by the Division of Chronic Disease Surveillance of the Korea Centers for Disease Control and Prevention to assess the health and nutritional status of the Korean civilian, noninstitutionalized population. It has been conducted annually since 2008, and approximately around 6,000 to 10,000 individuals are included in annual studies. A stratified, multistage probability sampling design was used for the selection of household units. Whole body and regional body composition were measured by DEXA (Discovery W, Hologic, Waltham, MA, USA) since 2008. Thus, many clinical investigations about sarcopenia and related clinical aspects have been conducted with this cohort.

The etiologies of sarcopenia are multifactorial. Diminishment of anabolic hormone, increased circulating levels of inflammatory cytokines, and lack of exercise with aging have been considered to be possible causative factors for those changes ${ }^{18)}$. Moreover, nutritional deficits have been also considered as one of the contributing factors for those changes ${ }^{19)}$. Kim et al ${ }^{20)}$ reported that vitamin D levels were significantly lower in sarcopenic subjects compared with nonsarcopenic subjects. In their study, sarcopenia was defined as an ASM adjusted weight index less than 2 SDs below the mean of healthy adults (20-40 years), and prevalence of sarcopenia in participants older than 50 years was $7.8 \%$. A report by Seo et al. ${ }^{21}$ ) showed that lower dietary calcium intake was significantly related to a greater risk of sarcopenia. Furthermore, studies using data from KNHANES have shown the cardiometabolic perspectives of sarcopenia indicating impaired metabolism and greater risk of 
cardiovascular disease (CVD). Chung et al. ${ }^{22)}$ reported a significant association between sarcopenic obesity and metabolic derangement indicated by a higher prevalence of metabolic syndrome. They also used weight-adjusted ASM as a skeletal muscle mass index, but they defined sarcopenia as ASM divided by weight (ASM/wt) that was less than 1 SD below the mean of healthy adults. Because of the relatively low threshold when defining sarcopenia, the prevalence of sarcopenia was found to be much higher at $42.0 \%$ in men and $42.7 \%$ in women aged 60 and older. The greater risks of diabetes and metabolic syndrome in sarcopenic subjects were similarity observed in a study reported by Moon ${ }^{23)}$, and the odds for diabetes and metabolic syndrome were signi- ficant in nonobese sarcopenic subjects. In this study, the ASM/wt index was used for assessing muscle mass, and ASM/wt less than 2 SDs below the mean of healthy adults was used when determining sarcopenia, and the rate of which was $4.26 \%$ in study subjects older than 20 years.

The greater risk of CVDs in subjects with sarcopenia has been observed in several studies. Chin et $\mathrm{al}^{2)}$ reported that CVD prevalence was significantly higher in the sarcopenic elderly than that of nonsarcopenic subjects. Weightadjusted ASM values less than 1 SD below the mean of healthy young adults were defined as indicating sarcopenia, and the overall prevalence of sarcopenia using this measure was 30.3\% in men and $29.3 \%$ in women older than 65 years. Moreover, sarcopenia was associated with a greater risk of high blood pressure in both obese and nonobese subjects, and ASM/wt values less than 1 SD below the mean of healthy young adults were used to categorize sarcopenia ${ }^{24)}$.

Importantly, skeletal muscles are essential for locomotion and mobility; thus, it is conceivable that sarcopenia might have dramatic consequences such as impaired performance ${ }^{1)}$. A significant association of sarcopenia with impaired physical activities was also demonstrated in KNAHNES data by Kim et al. ${ }^{25}$. Although the causal relationship has not yet been fully elucidated yet, a close relationship between sarcopenia and osteoporosis has been suggested, and a greater risk of osteoporosis in the elderly with sarcopenia than in the elderly without sarcopenia was observed in this cohort ${ }^{26)}$. However, in these two studies, muscle mass was analyzed using the ASM index adjusted by height squared, and an $\mathrm{ASM} / \mathrm{ht}^{2}$ of less than 2 SDs or 1 SD below the sex-specific normal mean for the young reference group was used as a definition for sarcopenia, respectively.

In this manner, both weight adjusted ASM index (ASM/ wt) and height squared adjusted ASM index (ASM/ht $\left.{ }^{2}\right)$ have been used for the definition of sarcopenia, and the prevalence of sarcopenia varies widely according to the method used.

\section{Studies in the Korean Longitudinal Study on Health and Aging}

The Korean Longitudinal Study on Health and Aging (KLoSHA) is an ongoing population-based observational study initiated in September 2005 for residents aged 65 or older in Seongnam city, one of the satellites of the Seoul Metropolitan district. Participants were selected using age and sex-stratified random sampling from a roster of people aged 65 and older in Seongnam, who were invited to participate in the study by letter and telephone. In total, 439 men and 561 women were ultimately included in the study. Whole body and regional body composition by DEXA (Lunar Corp., Madison, WI, USA) were included as a part of this study, and several measures in this cohort are related to sarcopenia.

We previously reported the prevalence of sarcopenia and the associations with risk of metabolic syndrome using data from this cohort ${ }^{17)}$. The cutoff values for sarcopenia were set as less than $1 \mathrm{SD}$ below the sex-specific mean for a young reference group, and both muscle mass parameters, $\mathrm{ASM} / \mathrm{wt}$ and $\mathrm{ASM} / \mathrm{ht}^{2}$, were used. Interestingly, the prevalence of sarcopenic obesity was $16.7 \%$ in men and $5.7 \%$ in women using the ASM/ht ${ }^{2}$ index; however, it was $35.1 \%$ in men and $48.1 \%$ in women by the $\mathrm{ASM} / \mathrm{wt}$ index. The ASM/wt index showed closer associations with metabolic impairments than an index based on $\mathrm{ASM} / \mathrm{ht}^{2}$. Our group recently reported an association of sarcopenia with increased mortality. For this study, we identified sarcopenic participants according to the EWGSOP recommendation, and two muscle mass indices, $\mathrm{ASM} / \mathrm{wt}$ and $\mathrm{ASM} / \mathrm{ht}^{2}$, were compared. As expected, the prevalence of sarcopenia was quite different according to the different muscle mass indices used, and the prevalence of sarcopenia was higher when muscle mass was assessed by ASM/ut than by ASM $/ \mathrm{ht}^{2}$. In men, the prevalence of sarcopenia was 8.8\% (25/284) using EWGSOP-ASM/ $\mathrm{ht}^{2}$ and $11.6 \%$ (33/284) using EWGSOP-ASM/wt. By contrast, the 
difference in the prevalence of sarcopenia using EWGSOP$\mathrm{ASM} / \mathrm{ht}^{2}$ and EWGSOP-ASM/wt was much greater in women: $8.8 \%(24 / 272)$ and $41.2 \%(112 / 272)$, respectively. Moreover, the adjusted hazard ratios of sarcopenia for mortality were 4.00 for ASM/ $\mathrm{ht}^{2}$ and 6.89 for ASM/wt in men. Interestingly, sarcopenia defined by these criteria was not associated with a greater risk of death in women. There are additional reports about the clinical consequences of sarcopenia regarding functional limitation or physical performance, and both ASM/wt and ASM/ht ${ }^{2}$ were used and compared in those studies ${ }^{27,28)}$.

\section{Data from Korean Genome Epidemiologic Study}

Two communities in Korea were selected for the Korean Genome Epidemiologic Study (KoGES) in 2001; the Ansung cohort represented a rural community, and the Ansan cohort an urban community. KoGES is an ongoing prospective study involving a biennial examination. Details of the KoGES and the method used have been described previously ${ }^{29)}$. In brief, a total of 10,038 subjects aged from 40 to 69 years were recruited $(5,020$ from a farming community, Ansung and 5,018 from an industrial community, Ansan). From the third visit of the participants in this study, whole body and regional body composition by DEXA (Lunar Corp.) were also included as a part of this study. Although there are as yet no published data, we could assess the DEXA data of this cohort and included the muscle parameters analyzed in this cohort in Table 1.

\section{Results from Korean Sarcopenic Obesity Study}

The Korean Sarcopenic Obesity Study (KSOS) is an ongoing prospective, observational cohort study initiated in September 2007. The KSOS was intended to examine the prevalence of sarcopenia and sarcopenic obesity in Korean adults and to evaluate the effects of sarcopenia and Sarcopenic Obesity on metabolic disorders and health outcomes. Using 2 SDs of ASM $/ \mathrm{ht}^{2}$ below reference values from young, healthy adults, the prevalence of sarcopenia and sarcopenic obesity were $4.1 \%$ and $0.8 \%$ respectively in older women ( $\geq 60$ years), and $6.3 \%$ and $1.3 \%$ respectively in older men ${ }^{30}$. By contrast, the prevalence of sarcopenia and sarcopenic obesity were $14.2 \%$ and $12.5 \%$ respectively in older women and $5.1 \%$ and $5.1 \%$ respectively in older men, using $2 \mathrm{SDs}$ below the $\mathrm{SM}^{30}{ }^{30}$. Interestingly, SMI values were significantly lower in patients with diabetes thab in those without diabetes ${ }^{31)}$. Moreover, patients with diabetes had a three times greater risk of sarcopenia (odds ratio, 3.06; 95\% confidence interval, 1.42 to 6.62) than subjects without diabetes in a multiple logistic regression analysis ${ }^{31)}$. As a single indicator of sarcopenic obesity, authors introduced the ratio of visceral fat to thigh muscle area (VMR) measured using computed tomography. VMR is significantly increased in subjects with metabolic syndrome and independently associated with metabolic syndrome ${ }^{32)}$. Multiple binary logistic regression analysis showed that a homeostasis model assessment of insulin resistance (HOMA-IR) and high sensitive C-reactive

Table 1. Mean values of anthropometric and sarcopenia indices in elderly Korean aged 65 years and over from representative Korean cohorts

\begin{tabular}{|c|c|c|c|c|c|c|c|c|c|c|}
\hline \multirow{2}{*}{$\begin{array}{c}\text { Study } \\
\text { population }\end{array}$} & \multirow{2}{*}{ No. } & \multirow{2}{*}{$\begin{array}{l}\text { Age } \\
(\mathrm{yr})\end{array}$} & \multirow{2}{*}{$\begin{array}{l}\text { Height } \\
(\mathrm{cm})\end{array}$} & \multirow{2}{*}{$\begin{array}{l}\text { Weight } \\
(\mathrm{kg})\end{array}$} & \multirow{2}{*}{$\begin{array}{c}\text { BMI } \\
\left(\mathrm{kg} / \mathrm{m}^{2}\right)\end{array}$} & \multirow{2}{*}{$\begin{array}{c}\text { ASM } \\
(\mathrm{kg})\end{array}$} & \multirow{2}{*}{$\begin{array}{c}\mathrm{ASM} / \mathrm{ht}^{2} \\
\left(\mathrm{~kg} / \mathrm{m}^{2}\right)\end{array}$} & \multirow{2}{*}{$\begin{array}{c}\text { ASM/wt } \\
(\%)\end{array}$} & \multicolumn{2}{|c|}{ Cutoffs for lowest quintile } \\
\hline & & & & & & & & & $\operatorname{ASM} / \mathrm{ht}^{2}\left(\mathrm{~kg} / \mathrm{m}^{2}\right)$ & ASM/wt (\%) \\
\hline \multicolumn{11}{|c|}{ KNHANES 2008-2010" } \\
\hline Men & 1,361 & $72.2 \pm 5.4$ & $164.5 \pm 5.9$ & $62.4 \pm 9.5$ & $23.0 \pm 2.9$ & $19.3 \pm 2.8$ & $7.1 \pm 0.8$ & $31.1 \pm 2.9$ & 6.43 & 28.8 \\
\hline Women & 1,923 & $72.9 \pm 5.9$ & $150.3 \pm 6.0$ & $54.6 \pm 9.2$ & $24.1 \pm 3.4$ & $13.4 \pm 1.9$ & $5.9 \pm 0.7$ & $24.8 \pm 2.9$ & 5.34 & 22.5 \\
\hline \multicolumn{11}{|l|}{$\mathrm{KLoSHA}^{\dagger}$} \\
\hline Men & 318 & $75.9 \pm 8.6$ & $164.4 \pm 6.2$ & $64.0 \pm 9.9$ & $23.6 \pm 3.2$ & $18.6 \pm 2.6$ & $6.9 \pm 0.8$ & $28.9 \pm 2.9$ & 6.19 & 26.4 \\
\hline Women & 315 & $76.0 \pm 8.8$ & $149.8 \pm 5.8$ & $54.2 \pm 9.4$ & $24.1 \pm 3.5$ & $12.8 \pm 2.3$ & $5.6 \pm 0.9$ & $23.3 \pm 4.5$ & 5.11 & 21.1 \\
\hline \multicolumn{11}{|l|}{$\mathrm{KoGES}^{\dagger}$} \\
\hline Men & 435 & $71.2 \pm 3.6$ & $163.7 \pm 5.6$ & $61.2 \pm 9.0$ & $22.8 \pm 3.1$ & $19.0 \pm 2.0$ & $7.1 \pm 0.7$ & $31.3 \pm 2.3$ & 6.49 & 29.5 \\
\hline Women & 284 & $71.0 \pm 3.5$ & $149.4 \pm 6.0$ & $54.1 \pm 8.6$ & $24.2 \pm 3.4$ & $13.2 \pm 1.6$ & $5.9 \pm 0.6$ & $24.5 \pm 1.8$ & 5.39 & 23.0 \\
\hline
\end{tabular}

Values are presented as mean \pm standard deviation.

BMI, body mass index; ASM, appendicular skeletal muscle mass; ht ${ }^{2}$, height squared (m); wt, weight (kg); KNAHNES, Korean National Health and Nutrition Examination Survey; KLoSHA, Korean Longitudinal Study on Health and Aging; KoGES, Korean Genome Epidemiology Study. "Hologic and ${ }^{\dagger}$ Luna dual energy X-ray absorptiometry system was used for measurement of muscle mass. 
protein were independently associated with sarcopenic obesity in women, while HOMA-IR and 25-hydroxyvitamin D levels were significant factors predicting sarcopenic obesity in men ${ }^{33)}$. Low muscle mass may be associated with different metabolic consequences according to body size phenotype, such as metabolically abnormal, but normal weight $t^{34}$. Furthermore, individuals with lower muscle mass exhibited greater risk of nonalcoholic fatty liver disease $^{35)}$. The longitudinal data of the KSOS demonstrated that visceral obesity at baseline was associated with future loss of skeletal muscle mass in Korean adults ${ }^{36)}$.

\section{PREVALENCE AND CUTOFFS FOR DEFINITION OF SARCOPENIA}

Both ASM/wt and ASM/ht ${ }^{2}$ indices have been used for assessing muscle mass, but the prevalence of sarcopenia varies according to which definition are used. The prevalence of sarcopenia was 9.3\% in Korean men and 0.2\% in women older than 65 years using the $\mathrm{ASM} / \mathrm{ht}^{2}$ index with data from KNHANES. However, the prevalence was $10.4 \%$ in men and $10.7 \%$ in women in the same study population using the $\mathrm{ASM} / \mathrm{wt}$ index ${ }^{37)}$. Variations in prevalence depending on the defining methods were similarly observed in other studies ${ }^{13,14,16)}$. Recently, several interventional modalities for preventing sarcopenia including nutritional support and promising drugs have been suggested $^{38)}$. However, characterizing which subjects could benefit most from those treatments should be a priority before applying treatment. Therefore, further studies to determine appropriate criteria and operational method to define vulnerable sarcopenic elderly people are warranted.

\section{CHANGES IN BODY COMPOSITION IN KOREANS}

Muscle mass change with aging shows quite distinguished patterns in Korean men and women ${ }^{37)}$. The ASM of men increases rapidly until 20 years of age and then decreases constantly until 90 years of age, with a more rapid loss after 60 years of age. However, ASM in women shows a slow increase until 20 years of age and then tends to remain constant until 50 years of age, followed by a decrease. ASM $/ \mathrm{ht}^{2}$ showed patterns similar to ASM in men, whereas the trend of ASM $/ \mathrm{ht}^{2}$ values among women was discordant with that of ASM, and it increased constantly until 60 years of age and then decreased. Changes with aging in ASM/wt values in men and women were also quite different, indicating a sharp increase until 20 years of age and then a gradual decrease until 30 years of age, followed by a gentle decline to 90 years of age in men, but a continuous decreased until 60 years of age followed by a slight rise in women (Fig. 1). According to these patterns, the mean values for healthy adults (20-40 years) represent the peak levels in each index for men. However, in women, the mean values for healthy adults $(20-40$ years) do not present the highest levels in either ASM or
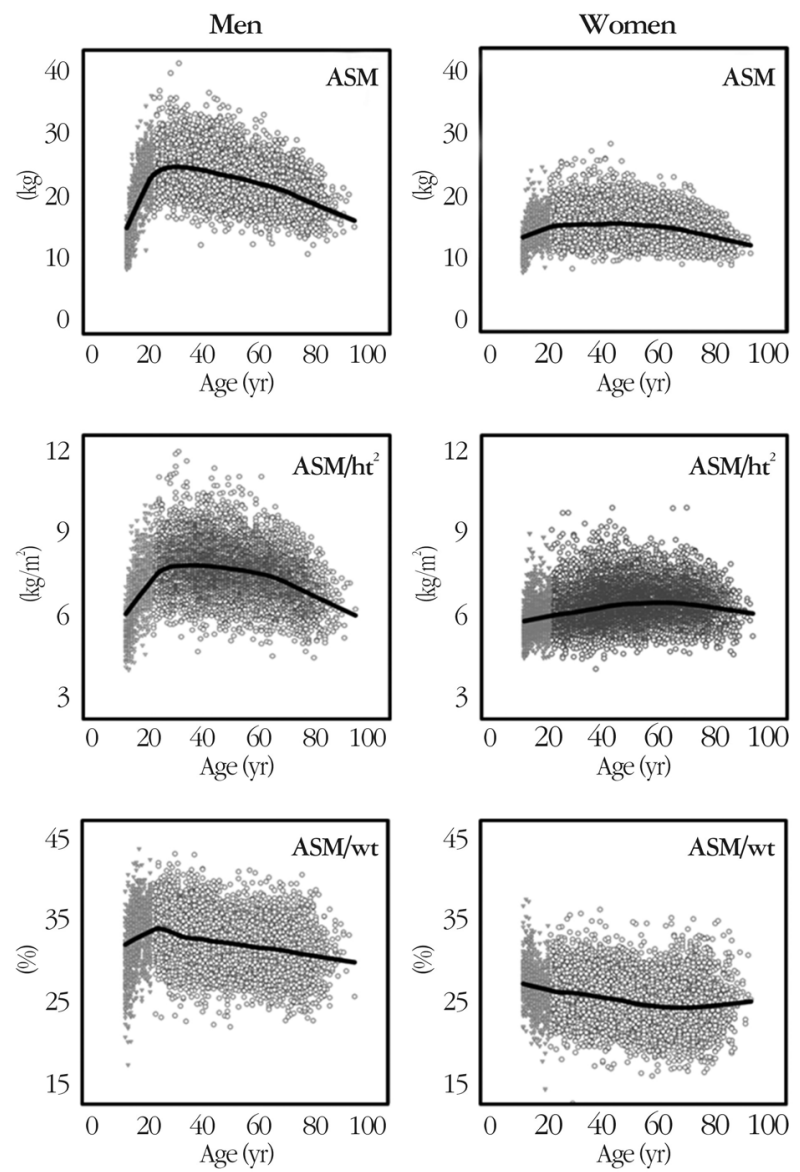

Fig. 1. Trends in ASM indices associated with aging in men and women using data from KNHANES 2008-2010. ASM, appendicular skeletal muscle mass; $\mathrm{ht}^{2}$, height squared (m); wt, weight (kg); KNAHNES, Korean National Health And Nutrition Examination Survey. 
$\mathrm{ASM} / \mathrm{ht}^{2}$. Based on these findings, whether or not the values in this age group (20-40 years) could be used as reference values is controversial.

Besides using the mean values of young reference groups, IWGS and EWGSOP recommend values of the lowest quintile in study populations as an alternative threshold for defining sarcopenia, ${ }^{5,7)}$. The values of each anthropometric and muscle mass parameter in the three cohorts mentioned are shown in Table 1. Although the numbers of elderly participants aged 65 years and older, included in each cohort study range from several hundred to several thousand, the values are quite similar in the same sexes. Therefore, it is conceivable that the method, using lowest quintile of a sex-specific study population, would better categorize elderly participants who have very low muscle mass in these study populations. Represen tatively, the cutoffs regarding lowest quintile of the elderly aged 65 years and older in KNHANES for ASM/ht ${ }^{2}$ or ASM/wt, is $6.43 \mathrm{~kg} / \mathrm{m}^{2}$ and $28.8 \%$ respectively in men and $5.34 \mathrm{~kg} / \mathrm{m}^{2}$ and $22.5 \%$ respectively in women (Table 1 ).

In conclusion, emerging evidence now suggests that sarcopenia is becoming more important in consideration of the health of the elderly and is closely related to various clinical consequences: not only functional limitation and impaired physical performance, but also metabolic derangement and cardiovascular risk. However, the operational definition of sarcopenia is still contentious. The prevalence of sarcopenia and its effects on various health conditions are different according to the methods used to define sarcopenia. While, the cutoff values for lowest quintile of a sex-specific study population are quite similar across the study population in Korea, and it would better categorize elderly participants who have very low muscle mass in these study populations. Representatively, the cutoffs regarding lowest quintile of the elderly aged 65 years and older in KNHANES for ASM/ $\mathrm{ht}^{2}$ or ASM/wt, is $6.43 \mathrm{~kg} / \mathrm{m}^{2}$ and $28.8 \%$ respectively in men and 5.34 $\mathrm{kg} / \mathrm{m}^{2}$ and $22.5 \%$ respectively in women. That is, validation of sarcopenia definitions that has been currently suggested is warranted to find the most clinically relevant criteria.

Recently, the Foundation of the National Institutes of Health Sarcopenia Project has proposed a novel operational method for measurement of muscle mass, and ASM standardized to BMI has been suggested ${ }^{39)}$. Future work should address the performance of this criterion within the relevant disease states in different ethnic groups including Koreans.

\section{SUMMARY}

Sarcopenia presents many negative health-related consequences including impaired energy homeostasis, risk of falls and cardiovascular disease, and subsequently higher mortality. It is becoming evident that sarcopenia has a negative impact on the healthy life of the elderly, and there have been also many investigations about sarcopenia in Korea. However, the prevalence of sarcopenia and its effects on various health conditions are different according to the methods used to define sarcopenia, ASM/wt or $\mathrm{ASM} / \mathrm{ht}^{2}$ in musle mass index, and less than 2 SDs below the mean of healthy adults or lowest quintile of a sex-specific study population in cutoffs for sarcopenia. Therefore, further studies are needed to reveal the most clinically relevant criteria for sarcopenia.

Conflict of Interest Disclosures: The researchers claim no conflicts of interest.

\section{REFERENCES}

1. Milte R, Crotty M. Musculoskeletal health, frailty and functional decline. Best Pract Res Clin Rheumatol 2014;28:395-410.

2. Chin SO, Rhee SY, Chon S, Hwang YC, Jeong IK, Oh S, et al. Sarcopenia is independently associated with cardiovascular disease in older Korean adults: the Korea National Health and Nutrition Examination Survey (KNHANES) from 2009. PLoS One 2013;8:e60119.

3. Kim JH, Lim S, Choi SH, Kim KM, Yoon JW, Kim KW, et al. Sarcopenia: an independent predictor of mortality in community-dwelling older Korean men. J Gerontol A Biol Sci Med Sci 2014;69:1244-52.

4. Lim S, Kwon SY, Yoon JW, Kim SY, Choi SH, Park YJ, et al. Association between body composition and pulmonary function in elderly people: the Korean Longitudinal Study on Health and Aging. Obesity (Silver Spring) 2011;19:631-8.

5. Cruz-Jentoft AJ, Baeyens JP, Bauer JM, Boirie Y, Cederholm T, Landi F, et al. Sarcopenia: European consensus on definition and diagnosis: Report of the European Working Group on Sarcopenia in Older People. Age Ageing 2010;39:412-23.

6. Chen LK, Liu LK, Woo J, Assantachai P, Auyeung TW, Bahyah KS, et al. Sarcopenia in Asia: consensus report of the Asian Working Group for Sarcopenia. J Am Med Dir 
Assoc 2014;15:95-101.

7. Fielding RA, Vellas B, Evans WJ, Bhasin S, Morley JE, Newman $\mathrm{AB}$, et al. Sarcopenia: an undiagnosed condition in older adults. Current consensus definition: prevalence, etiology, and consequences. International working group on sarcopenia. J Am Med Dir Assoc 2011;12:249-56.

8. Janssen I, Heymsfield SB, Ross R. Low relative skeletal muscle mass (sarcopenia) in older persons is associated with functional impairment and physical disability. J Am Geriatr Soc 2002;50:889-96.

9. Baumgartner RN, Koehler KM, Gallagher D, Romero L, Heymsfield SB, Ross RR, et al. Epidemiology of sarcopenia among the elderly in New Mexico. Am J Epidemiol 1998;147: 755-63.

10. Genaro Pde S, Pinheiro Mde M, Szejnfeld VL, Martini LA. Secondary hyperparathyroidism and its relationship with sarcopenia in elderly women. Arch Gerontol Geriatr 2015;60: 349-53.

11. Di Monaco M, Castiglioni C, De Toma E, Gardin L, Giordano S, Di Monaco R, et al. Presarcopenia and sarcopenia in hip-fracture women: prevalence and association with ability to function in activities of daily living. Aging Clin Exp Res. 2015 Jan 10 [Epub]. http://dx.doi.org/10.1007/s40520-014-0306-z.

12. Lee WJ, Liu LK, Peng LN, Lin MH, Chen LK; ILAS Research Group. Comparisons of sarcopenia defined by IWGS and EWGSOP criteria among older people: results from the I-Lan longitudinal aging study. J Am Med Dir Assoc 2013;14:528. e1-7.

13. Batsis JA, Barre LK, Mackenzie TA, Pratt SI, Lopez-Jimenez F, Bartels SJ. Variation in the prevalence of sarcopenia and sarcopenic obesity in older adults associated with different research definitions: dual-energy X-ray absorptiometry data from the National Health and Nutrition Examination Survey 1999-2004. J Am Geriatr Soc 2013;61:974-80.

14. Scott D, Hayes A, Sanders KM, Aitken D, Ebeling PR, Jones G. Operational definitions of sarcopenia and their associations with 5-year changes in falls risk in community-dwelling middle-aged and older adults. Osteoporos Int 2014;25:187-93.

15. Kim JH, Hwang Bo Y, Hong ES, Ohn JH, Kim CH, Kim $\mathrm{HW}$, et al. Investigation of Sarcopenia and its association with cardiometabolic risk factors in elderly subjects. J Korean Geriatr Soc 2010;14:121-30.

16. Newman AB, Kupelian V, Visser M, Simonsick E, Goodpaster B, Nevitt M, et al. Sarcopenia: alternative definitions and associations with lower extremity function. J Am Geriatr Soc 2003;51:1602-9.

17. Lim S, Kim JH, Yoon JW, Kang SM, Choi SH, Park YJ, et al. Sarcopenic obesity: prevalence and association with metabolic syndrome in the Korean Longitudinal Study on Health and Aging (KLoSHA). Diabetes Care 2010;33:1652-4. 18. Ali S, Garcia JM. Sarcopenia, cachexia and aging: diagnosis, mechanisms and therapeutic options - a mini-review. Gerontology 2014;60:294-305.

19. Malafarina V, Uriz-Otano F, Iniesta R, Gil-Guerrero L. Sarcopenia in the elderly: diagnosis, physiopathology and treatment. Maturitas 2012;71:109-14.

20. Kim MK, Baek KH, Song KH, Il Kang M, Park CY, Lee WY, et al. Vitamin D deficiency is associated with sarcopenia in older Koreans, regardless of obesity: the Fourth Korea National Health and Nutrition Examination Surveys (KNHANES IV) 2009. J Clin Endocrinol Metab 2011;96: 3250-6.

21. Seo MH, Kim MK, Park SE, Rhee EJ, Park CY, Lee WY, et al. The association between daily calcium intake and sarcopenia in older, non-obese Korean adults: the fourth Korea National Health and Nutrition Examination Survey (KNHANES IV) 2009. Endocr J 2013;60:679-86.

22. Chung JY, Kang HT, Lee DC, Lee HR, Lee YJ. Body composition and its association with cardiometabolic risk factors in the elderly: a focus on sarcopenic obesity. Arch Gerontol Geriatr 2013;56:270-8.

23. Moon SS. Low skeletal muscle mass is associated with insulin resistance, diabetes, and metabolic syndrome in the Korean population: the Korea National Health and Nutrition Examination Survey (KNHANES) 2009-2010. Endocr J 2014;61: 61-70.

24. Han K, Park YM, Kwon HS, Ko SH, Lee SH, Yim HW, et al. Sarcopenia as a determinant of blood pressure in older Koreans: findings from the Korea National Health and Nutrition Examination Surveys (KNHANES) 2008-2010. PLoS One 2014;9:e86902.

25. Kim SH, Kim TH, Hwang HJ. The relationship of physical activity (PA) and walking with sarcopenia in Korean males aged 60 years and older using the Fourth Korean National Health and Nutrition Examination Survey (KNHANES IV-2, 3), 2008-2009. Arch Gerontol Geriatr 2013;56:472-7.

26. Huh JH, Song MK, Park KH, Kim KJ, Kim JE, Rhee YM, et al. Gender-specific pleiotropic bone-muscle relationship in the elderly from a nationwide survey (KNHANES IV). Osteoporos Int 2014;25:1053-61.

27. Kim JH, Choi SH, Lim S, Yoon JW, Kang SM, Kim KW, et al. Sarcopenia and obesity: gender-different relationship with functional limitation in older persons. J Korean Med Sci 2013;28:1041-7.

28. Kim KE, Jang SN, Lim S, Park YJ, Paik NJ, Kim KW, et al. Relationship between muscle mass and physical performance: is it the same in older adults with weak muscle strength? Age Ageing 2012;41:799-803.

29. Lim S, Jang HC, Lee HK, Kimm KC, Park C, Cho NH. A rural-urban comparison of the characteristics of the metabolic syndrome by gender in Korea: the Korean Health and Genome Study (KHGS). J Endocrinol Invest 2006;29:313-9. 
30. Kim TN, Yang SJ, Yoo HJ, Lim KI, Kang HJ, Song W, et al. Prevalence of sarcopenia and sarcopenic obesity in Korean adults: the Korean sarcopenic obesity study. Int J Obes (Lond) 2009;33:885-92.

31. Kim TN, Park MS, Yang SJ, Yoo HJ, Kang HJ, Song W, et al. Prevalence and determinant factors of sarcopenia in patients with type 2 diabetes: the Korean Sarcopenic Obesity Study (KSOS). Diabetes Care 2010;33:1497-9.

32. Lim KI, Yang SJ, Kim TN, Yoo HJ, Kang HJ, Song W, et al. The association between the ratio of visceral fat to thigh muscle area and metabolic syndrome: the Korean Sarcopenic Obesity Study (KSOS). Clin Endocrinol (Oxf) 2010; 73:588-94.

33. Kim TN, Park MS, Lim KI, Choi HY, Yang SJ, Yoo HJ, et al. Relationships between sarcopenic obesity and insulin resistance, inflammation, and vitamin D status: the Korean Sarcopenic Obesity Study. Clin Endocrinol (Oxf) 2013;78: 525-32.

34. Kim TN, Park MS, Yang SJ, Yoo HJ, Kang HJ, Song W, et al. Body size phenotypes and low muscle mass: the Korean sarcopenic obesity study (KSOS). J Clin Endocrinol Metab
2013;98:811-7.

35. Hong HC, Hwang SY, Choi HY, Yoo HJ, Seo JA, Kim SG, et al. Relationship between sarcopenia and nonalcoholic fatty liver disease: the Korean Sarcopenic Obesity Study. Hepatology 2014;59:1772-8.

36. Kim TN, Park MS, Ryu JY, Choi HY, Hong HC, Yoo HJ, et al. Impact of visceral fat on skeletal muscle mass and vice versa in a prospective cohort study: the Korean Sarcopenic Obesity Study (KSOS). PLoS One 2014;9:e115407.

37. Kim KM, Lim S, Choi SH, Kim JH, Shin CS, Park KS, et al. Cardiometabolic implication of sarcopenia: The Korea National Health and Nutrition Examination Study (KNHANES) 2008-2010. IJC Metab Endocr 2014;4:63-9.

38. Wakabayashi H, Sakuma K. Comprehensive approach to sarcopenia treatment. Curr Clin Pharmacol 2014;9:171-80.

39. McLean RR, Shardell MD, Alley DE, Cawthon PM, Fragala MS, Harris TB, et al. Criteria for clinically relevant weakness and low lean mass and their longitudinal association with incident mobility impairment and mortality: the foundation for the National Institutes of Health $(\mathrm{FNIH})$ sarcopenia project. J Gerontol A Biol Sci Med Sci 2014;69:576-83. 